\title{
Quadrilateral panelization of freeform surface structures
}

\author{
Aysu Berk $^{\mathrm{a}, *}$, Harry Giles ${ }^{\mathrm{b}}$ \\ a Bilkent University, Faculty of Art, Design and Architecture, Department of Architecture, Ankara, Turkey \\ b University of Michigan, Taubmann College of Architecture and Urban Planning, Ann Arbor, USA
}

\section{A R T I C L E I N F O}

Article history:

Received 21 July 2015

Received in revised form 14 December 2016

Accepted 8 January 2017

Available online 18 January 2017

\section{Keywords:}

Freeform surfaces

Quadrilateral discretization

Non-planarity

Warping

\begin{abstract}
A B S T R A C T
Freeform surfaces need to be discretized into manageable sized panels so that the surface can be fabricated in smaller pieces that are then assembled on site. This discretization process comprises numerous challenges; including not only the aesthetic and spatial concerns, but also the structural and constructional needs. The mesh generated has to fit to the surface, without distorting the form; meanwhile the panels have to preserve their planarity in order to prevent any brittle failure due to warping. This study proposes a unique approach to quadrilateral discretization by incorporating the form, material capacities and fabrication challenges in order to obtain a structure that can be realized and constructed.

In this paper, quadrilateral discretization of freeform surfaces is studied with the focus of material properties. The possibility of utilizing quadrilateral meshes with limited non-planarity is explored. The capacity of materials against warping is calculated, by structural experiments and simulations to obtain the limiting values that are integrated into design as tolerance of panels to non-planarity. Consequently, the amount of non-planarity becomes a parameter that needs to be considered during the generation of the quadrilateral mesh in order to generate an optimum surface discretization.
\end{abstract}

(c) 2017 Elsevier B.V. All rights reserved.

\section{Introduction}

Freeform surface structures can be distinguished from other types of structures by their unique, amorphous shapes, smooth continuous lines, and complex geometries [1]. In contrast to traditional structural design of horizontal beams and vertical columns, freeform surface structures do not have a separate structural system; instead the surface is designed to function as the main structural system. Therefore, the integration of the structural system with the amorphous form causes complexities, not only in the design process, but also in the realization and construction of the structure. These problems of design and fabrication of freeform surfaces have always been an important issue from the early examples of freeform surfaces in 1920s till today's more contemporary examples.

Heinz Isler (1926-2009), a pioneering name on lightweight concrete shells, used physical models to generate efficient forms; shells that carried the required load with the minimum possible material [2]. As concrete could be poured into the desired form; Isler's shells could be fabricated and constructed. However, the challenge was to obtain formworks with the complex geometry. Moreover, his method was feasible to construct in concrete but did not work with other materials.

Due to these limitations, discrete systems had been considered, which provided the application of other materials than concrete. A

\footnotetext{
* Corresponding author.

E-mail address: aysu.berk@bilkent.edu.tr (A. Berk).
}

common application has been the grid shell, where the surface is made up of discrete structural members, mostly orthogonal to each other, with panels fitting in between them. Grid shells have been considered in the design of freeform surfaces in order to overcome the fabrication problems [3]. These discrete surfaces have not only offered advantages for fabrication and construction, but have also provided spacious designs due to the use of transparent materials for the panels [3-5]. The main structural framing is usually selected to be steel, whereas glass has been preferred for the panels because of both its strength and transparency. Because of the brittle property of glass, panels have mostly been used as planar sheets. In some rare cases, glass was utilized as bent or curved sheet; i.e. spherical dome of the swimming arena in Neckarsulm (Fig. 1a), where the glass sheets were manufactured with curvature [6] or in the case of the German Historical Museum roof, where the glass plates were manufactured flat and then bent and warped during the assembly to obtain the continuous smooth roof system required [7] (Fig. 1b).

Grid shells have mostly been applied to surfaces with regular geometries (translational, rotational, etc.). However, when the form is organic, not generated by simple geometric rules (not a derivative of a translational, rotational surfaces), the panelization (discretization) process becomes a challenge. The panels obtained by the discretization have problems either with size, form or structural strength. Different patterns (triangulations, quadrilaterals, or hexagons) of discretization have been applied on freeform surfaces, resulting in meshes with different advantages and limitations. 


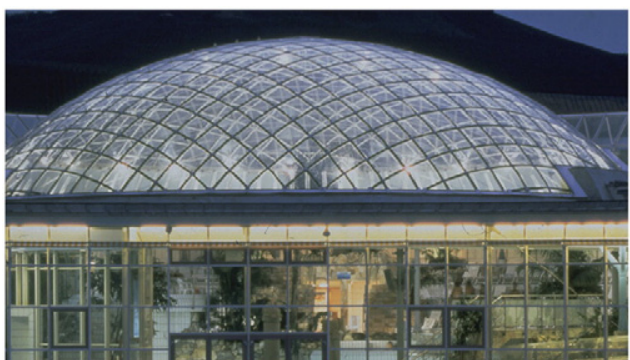

a

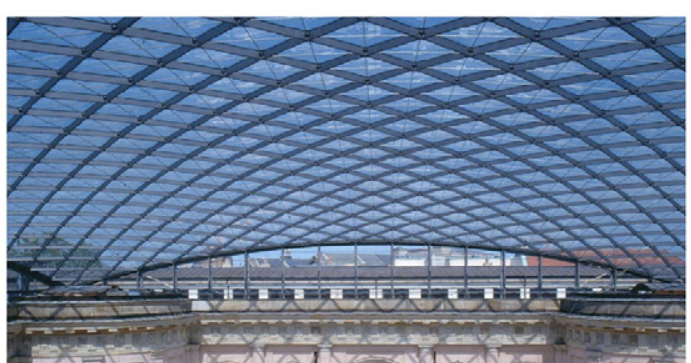

b

Fig. 1. (a) Swimming Arena in Neckarsulm [7]. (b) German Historical Museum [7].

Triangulations have been the most common pattern in order to discretize freeform surfaces not only because of their stable geometry, but also aesthetically satisfying organization and the way they generate planar surfaces [8]. However, automation of the construction process is difficult due to the non-standardization of the nodes in triangulation. Each node needs to be custom-designed as six members per node causing a complex joint assembly.

To prevent the problems encountered with triangulation, quadrilateral panels are considered where 4 members join at one node in contrast to 6 members in triangulation. With less number of members connecting at each node, design and construction of these joints become less complicated. In addition, quadrilateral meshes generate a less dense network of members that provides a more spacious feeling underneath. However, the major problem for quadrilateral discretization is that not every quadrilateral panel is planar. Therefore, unless all the panels are fully planar, there is the problem of sudden fracture of brittle materials, such as glass that is commonly used for discrete surface panelization. That is why quadrilateral discretization focuses on the planarity of panels while generating the mesh.

Various methods for the generation of planar quadrilateral meshes have been studied. Some propose triangulation on a surface as the initial step where they can be re-meshed to generate quadrilaterals with planar faces [9-11]. Other studies use methods and algorithms that generate quadrilaterals on the surface directly $[9,12,13]$. Alliez et al. [9] had a geometric approach to this problem where they proposed an algorithm based on lines of principal curvatures, ${ }^{1}$ which intersect each other at right angles and generate approximately planar panels by the intersection points of these curvature lines [8]. Their study projects a great potential for the freeform surfaces to be mapped with these principal curvatures and obtaining planar quadrilateral panels. However, when the method is analysed considering its practical applications, it is observed that panels generated by the principal curvature lines do not have constant or similar mesh size, especially when they are applied on freeform surfaces, which have dramatic changes of surface curvature, resulting a non-homogeneous mesh distribution (Fig. 2a) [14]. These uneven mesh sizes cause problems at the fabrication process. Panels that are close to umbilic points ${ }^{2}$ or high curvature points are so small that they can neither be materialized nor physically constructed (Fig. $2 \mathrm{~b})$. At the points where principal curvatures do not solve the singularities, non-quadrilateral panels need to be used in order to obtain the continuity of the generated mesh.

\footnotetext{
${ }^{1}$ Principal Curvature Lines: At any specific point on a surface, infinite number of plains that are normal to the surface at that point can be drawn, corresponding to a specific normal curve that is a part of a circle that defines the surface curvature at that point. Among these infinite sections and curvature lines, there occur a unique set of minimum and maximum curvature lines at each point that are called as principal curvature lines. They are represented by $\mathrm{k}_{1}$ and $\mathrm{k}_{2}$ respectively where $\mathrm{k}=1$ /radius.

2 Umbilic Point (Umbilics): Points on a surface where there are no unique maximum or minimum, but infinite principal curvature lines. The surface is either flat or spherical at that point.
}

Another method to generate quadrilateral meshing on freeform surfaces has been an optimization algorithm, Evolute Pro [15], that has constraints, such as planarity, surface closeness, fairness of curvature, etc. where many of these constraints can be applied simultaneously with the appropriate weights assigned to them. Panels are generated either as triangulated or quadrilateral. The advantage of this tool is its ability to optimize a homogeneously distributed quadrilateral mesh on the surfaces considering both the planarity of the panels and their best fit to the original surface. However, the size and/or number of the generated panels are determined automatically. Therefore, the designer does not have full control over the final mesh design. Moreover, with the increase of complexity of the surface, the absolute planarity is not achieved completely for all panels.

These aforementioned studies demonstrate that obtaining freeform surface discretization with planar quadrilaterals has various challenges; either problems to obtain absolute planarity of the panels, or to control the distribution of panels (both size and number) through these surfaces. This study focuses on the planarity issue of these panels and questions the limits of planarity in order to help the meshing process. In recent works, panels have been designed with the obligation of being planar and some precision is accepted for the planarity measurements with no consideration of the material properties [16-18]. However, the tolerance of each material to non-planarity is different and this tolerance can be used in the design process to obtain an optimized design.

\section{The methodology}

This study demonstrates the utilization of non-planar quadrilateral panels for the discretization of free form surfaces. The first step is to determine the tolerance of materials for non-planarity by structural analyses. These analyses are conducted through the steps of choosing a designated material, building the appropriate set-up, and to conduct the experiments. Then, a parametric relationship is derived between the panel sizes and the deformation limits, in order to calculate the limiting condition for any panel size. Finally, the generated quadrilateral mesh is analysed, and the curvature occurring at the panels is compared with the limits of the selected material. If the selected material is not capable of resisting to that curvature, then either the material can be changed or the surface can be re-discretized.

This study particularly aims to demonstrate the complete methodology more than to conduct a structural analysis study. The focus is, therefore, more on the process and the idea of utilizing non-planarity into design than the detailed study of structural analyses. Results obtained in this study are case-specific to demonstrate the application of the methodology that can be used in further studies as a foundation. For this study, analyses are conducted with one selected material and the experiment set-up is built for this case in the lab.

\subsection{The material}

In this study, glass is selected as the designated material experiment material; because glass has been a common material for freeform 




a

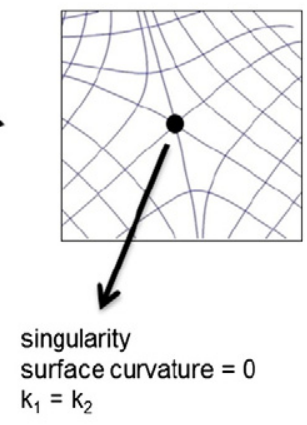

b

Fig. 2. (a) Principal curvature line distribution. (b) Umbilic points.

surface panelization. In addition to its strength, glass provides a good spatial quality to continuous surfaces due to its transparency. However, it should also not be forgotten that glass is a brittle material; therefore warping is a critical issue for this material.

Glass is a strong material in compression, but weak in tension [19]. Due to its brittle properties, glass fails suddenly with little warning. In order to enhance glass for structural reasons, heat treatment is applied. By heating the glass to high temperatures and cooling it rapidly, the surface cools quicker than the inner part and generates a surface stress different to the inner stress, which causes pre-compression of the glass [19]. The pre-compression generated can give extra tensile capacity to the material. In Fig. 3, the increase in the capacity can be seen as double for heat strengthened and four times higher for fully tempered glasses compared to annealed glass. Because of these reasons, during the experiments, fully tempered (structural) glass was used.

\subsection{The experiment set-up}

The experiments are conducted on $915 \mathrm{~mm}$ by $915 \mathrm{~mm}$ (36" by 36") fully tempered glass panels with $3 \mathrm{~mm}$ thickness. The glass is framed with $3 \mathrm{~mm}$ thick steel framing. Neoprene rubber gaskets, thickness of
$3 \mathrm{~mm}$, are used in between the steel frames and the glass sheet in order to allow the glass to rotate mildly under the load, which is a common application for glass frames. To make the edges of this glass-steel sandwich stiffer, aluminum hollow tubes are attached to the four edges of the frame (Fig. 4). Strain gauges are used to measure the directional strain at specific points. The locations of strain gauges are determined with respect to where maximum and minimum strain (and stress) is expected to occur based on the results of initial simulations.

The analyses are divided into two phases: simply supported bending analysis and the non-planarity (warping) analysis.

The first phase is a simply supported bending test where quadrilateral panel is supported by its two parallel edges and a uniform load is applied on the mid span (Fig. 5). The aim of the simple bending test is to observe the basic behaviour of the panel under three-point loading, to calibrate the system, and to calculate the material properties of the glass sheet used. $915 \mathrm{~mm}$ by $915 \mathrm{~mm}$ panel is supported by knifeedge supports on opposite sides. Load is equally distributed across the length of the panel in the mid span. One strain gauge is located at the midpoint of the sheet to record the maximum strain. During the simple bending experiment, the initial deformation due to the glass self-weight is taken as negligible since it is quite small with respect to the deflection under the load.



Fig. 3. The stress capacity of glass with different heat-treatments. 


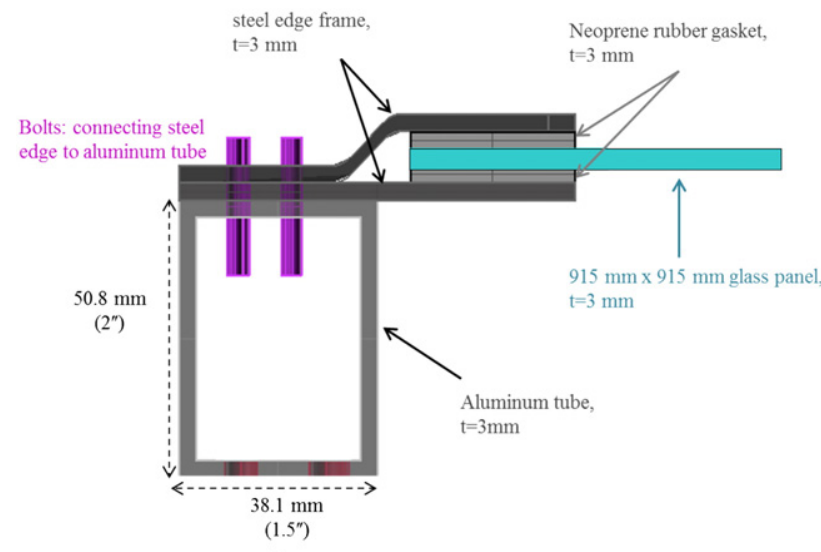

a

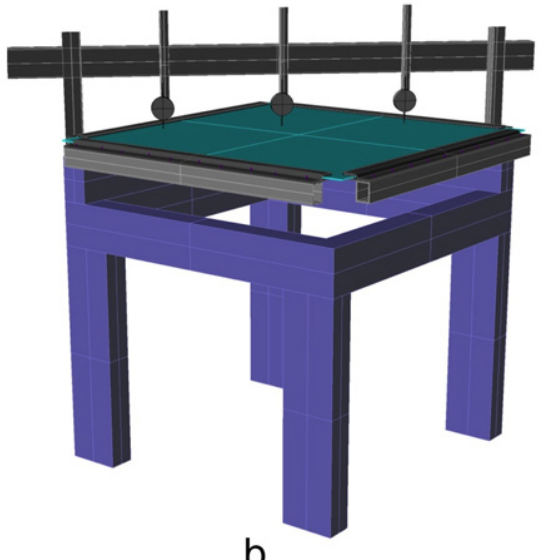

b

Fig. 4. The experiment set-up. (a) The cross section. (b) The experiment table.

The second phase of the experiment is the non-planarity (warping) analysis where a same sized sheet is supported by its two adjacent edges with the other two edges left free and the load is hung from the free corner of the panel (Fig. 6). The purpose of this phase is to determine the limiting deformation, therefore the limiting curvature of the sheet. For the warping test, strain gauges are located diagonally at four critical points; two on one side and other two on the other side of the glass sheet (Fig. 6). During this phase, the initial deformation due to the self-weight of the sheet (including the frames) cannot be neglected, since they generate a significant amount of pre-deformation before the load is applied.

In order to support the results obtained by the physical experiments, the same conditions are modeled by computer simulations, with the frame around the glass sheet simplified to a single stiffening edge instead of the real case of an aluminum tube and neoprene bands [14].

\subsubsection{Simply supported bending analysis}

Before the non-planarity analysis, initial tests and calculations were conducted to calibrate the experimental set-up, to observe the behaviour of the material under simple loading, and to compare the results with the computer analysis.

Simply supported bending analysis of a panel sheet is calculated, taking a simply supported beam as the base. The panel is assumed to behave as a beam with a point load in the middle as seen in Fig. 7. The maximum deflection for the panel is calculated where $\Delta$ is the mid (maximum) deflection of the panel, $\mathrm{P}$ is the applied concentrated load, $\mathrm{L}$ is the span between the supports, $\mathrm{E}$ is the young's modulus of the material used and $\mathrm{I}$ is the moment of inertia.

The concentrated point load is determined as $222.4 \mathrm{~N}$ ( $50 \mathrm{lb}$ ) within the limitation of the study. The clear span is measured as $845 \mathrm{~mm}(\mathrm{~L})$ and Young's Modulus for the material is taken as $70 \mathrm{GPa}$. For the calculation of the moment of inertia, the width of the panel (b), is taken as $915 \mathrm{~mm}$, whereas the thickness of the sheet is used for the height of the section (h), that is $3 \mathrm{~mm}$. For the maximum stress calculation, c, the maximum vertical distance from neutral axis, is taken as half of the thickness of the sheet $(1.5 \mathrm{~mm})$.

Moment of inertia $: I=\frac{1}{12} b t^{3}=\frac{1}{12}(915)(3)^{3}=2058.75 \mathrm{~mm}^{4}$

Maximum Moment : $M=\frac{\mathrm{PL}}{4}=\frac{(222.4)(0.845)}{4}=46.98 \mathrm{~N} \cdot \mathrm{m}$

Maximum Stress : $\sigma=\frac{M c}{I}=\frac{(46.98)\left(1.5 \times 10^{-3}\right)}{2058.75 \times 10^{-12}}=34.24 \mathrm{MPa}$

Maximum Deformation : $\Delta=\frac{\mathrm{PL}^{3}}{48 \mathrm{EI}}=\frac{(222.4)(0.845)^{3}}{48\left(70 \times 10^{9}\right)\left(2058.75 \times 10^{-12}\right)}=19.4 \mathrm{~mm}$

The theoretical calculations above show that the maximum deformation under this loading is expected to be $19.4 \mathrm{~mm}$ with a maximum stress of 34.24 MPa. The similar set-up is simulated with the computer program and the same load is applied on the glass sheet. Maximum deflection resulted from the simulations is $20.6 \mathrm{~mm}$ and maximum stress is $40 \mathrm{MPa}$ as shown below in Fig. 8 .

The results obtained from the simulation fit the analytic calculations and demonstrate that the fully tempered glass can carry the load of 222.4 N throughout this experiment. Therefore, the same conditions


Fig. 5. Simple bending test. 

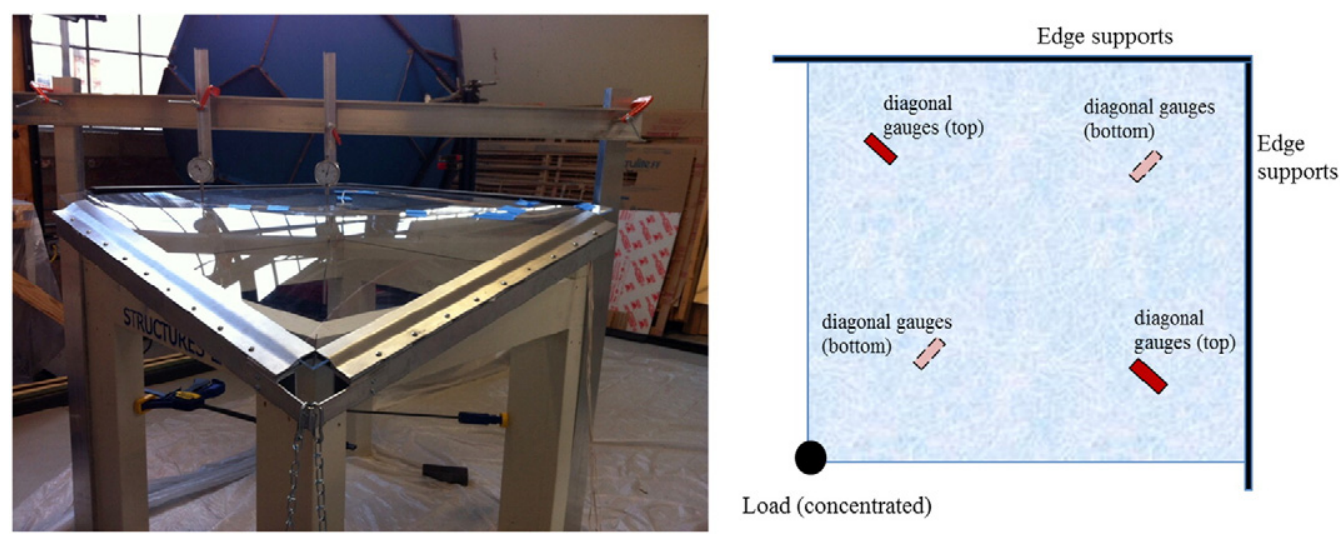

Load (concentrated)

Fig. 6. Warping test.
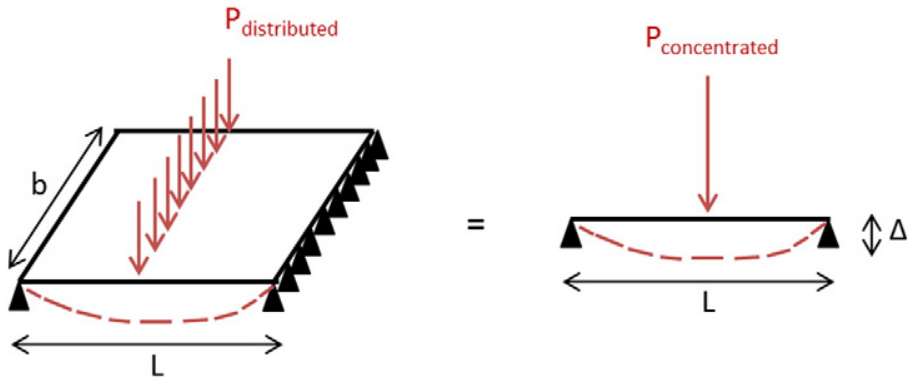

$$
\Delta=\frac{\mathrm{PL}^{3}}{48 \mathrm{EI}}
$$

Fig. 7. Simple bending - deformation schema for the panel under linear load.

are applied on the experimental set-up. The stress-strain graphic is drawn by the values recorded by the logger as shown in Fig. 9.

Using the recorded values of strain gauges $\left(\Delta=0.578^{\prime \prime}=14.68 \mathrm{~mm}\right.$ and $\varepsilon=4 \times 10^{-4}$ ), the Young's Modulus for the material is calculated as:

$E=\frac{P L^{3}}{48 \Delta \mathrm{I}}=\frac{(222.4)(0.845)^{3}}{48\left(14.68 \times 10^{-3}\right)\left(2058.75 \times 10^{-12}\right)}=92.5 \mathrm{GPa}$

Then, the maximum stress is calculated as shown below:

$\sigma=E \times \varepsilon=(92.5)\left(4 \times 10^{-4}\right)=0.037 \mathrm{GPa}=37 \mathrm{MPa}$

Having obtained all these results from three different methods of inspection (Theoretical, simulations and experiments), Table 1 is prepared to compare and contrast the results.
The differences between the stresses are partially due to the material properties that vary between theory and practice. It is possible to use the material properties obtained from the experiments back into the simulations to obtain more precise results but for the purpose of this study, the theoretical values are accepted to be enough.

The maximum deformation measured during the physical test is observed to be less than the theoretical calculations. This difference indicates that along with the inconsistencies of materials, membrane stresses generated on the glass sheet reduce the amount of deformation. This observation shows that non-linear analysis should be considered during these investigations.

In conventional structural analyses, the calculations are done based on linear behaviour. However, when the deformation exceeds half of the thickness of the panel, the stress-strain relationship cannot be taken linearly [19]. In this test, the amount of deformation is expected to exceed half of the thickness of the panel; therefore non-linear

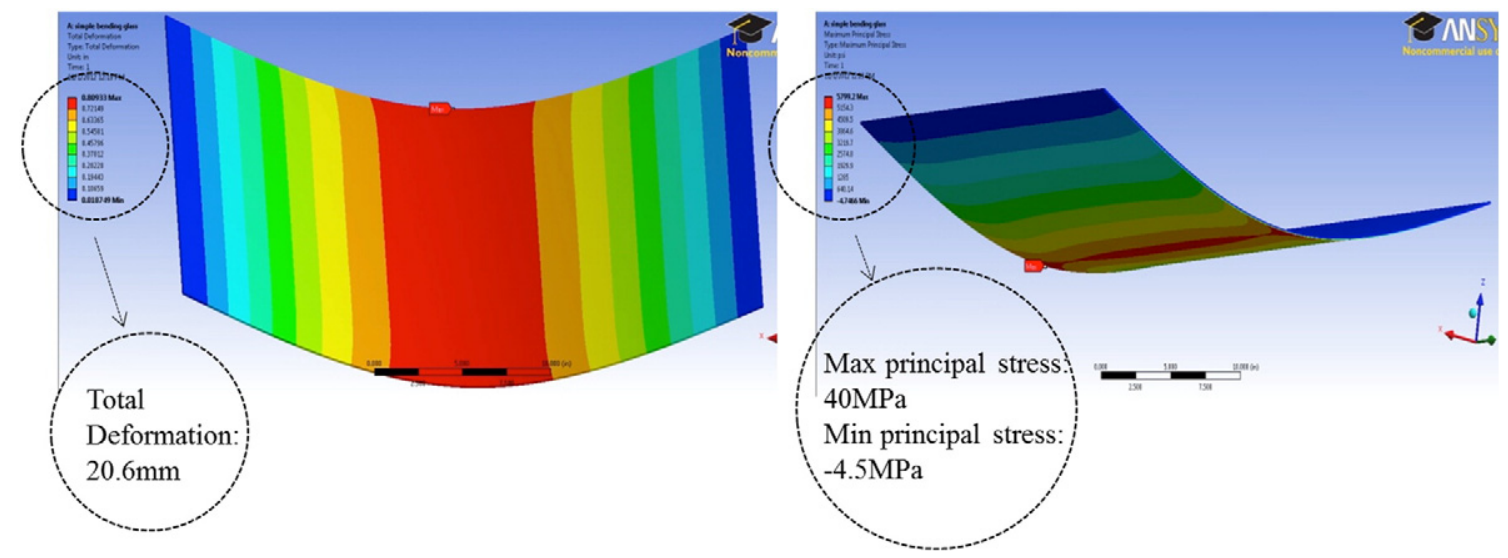

Fig. 8. The simple bending analysis results for the glass sheet under the load. 

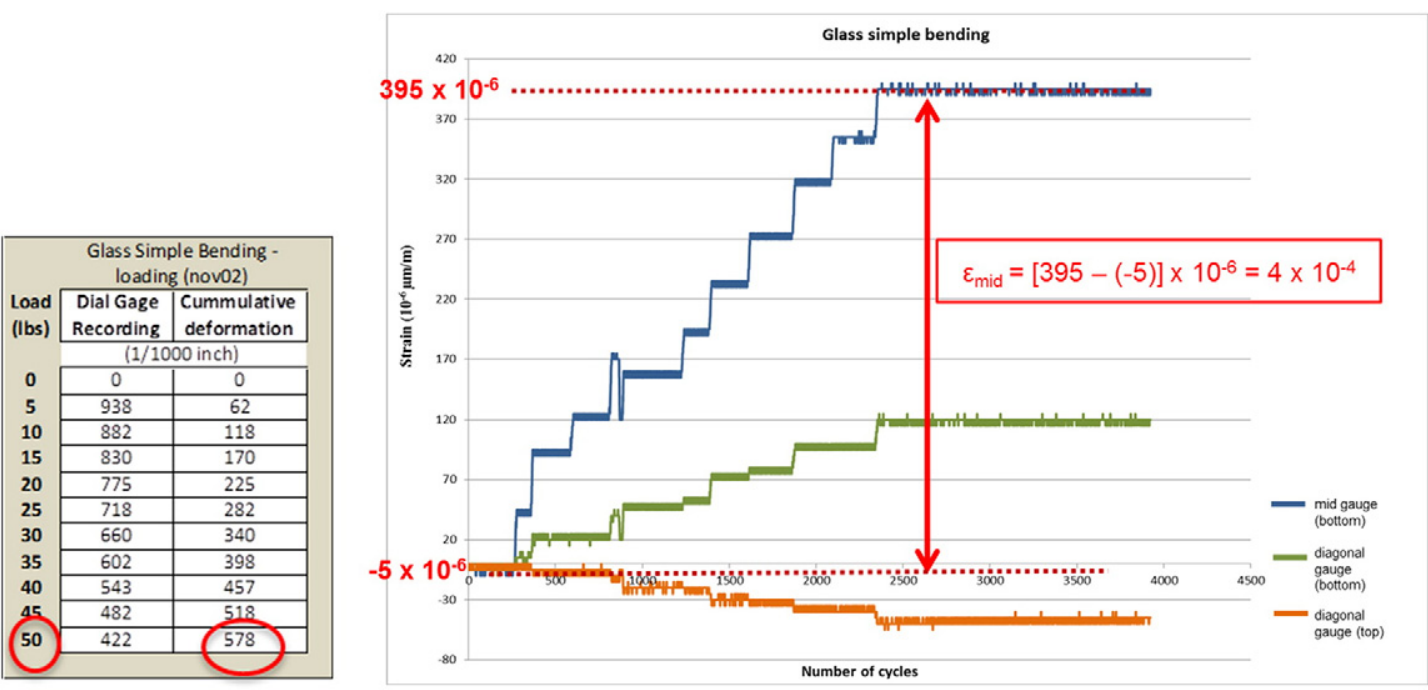

Fig. 9. The time history plot for the simple bending test.

(large deflection) analyses are preferred. During non-linear deformation, due to the edge restraints, axial stresses are generated that are called membrane stresses. They can be either in tension or compression, depending on the type of constraint on the edges. The total stress on a section must be calculated as the sum of the bending stress and the membrane stresses.

\subsubsection{Warping analysis}

As mentioned before, the initial deformation due to the self-weight of the glass sheet is not neglected for this analysis. In order not to experience a sudden failure of the glass under its own weight, the glass is left to carry its weight in discrete intervals. It is observed that the sheet is deformed approximately $55 \mathrm{~mm}$ (2.20") under self-weight (Fig. 10). The experimentation is continued by loading the glass in the increments


the critical points (the principal stress locations) are calculated using the recordings of strain values and the maximum deformation is recorded by the dial gauges.

It is observed from the strain gauge readings that on the bottom surface of the sheet, tension stresses increase to a certain value during the loading and then start to decrease due to the membrane stresses. The strain values, therefore the stresses on the bottom surface, become zero when the maximum deflection occurs. On the other hand, on the top surface of the sheet, the strain gauge values increase through the loading, causing to generate compressive stresses (Fig. 10).

The surface stresses at the maximum deformation can be calculated by the generalized form of Hooke's law:

$\sigma=\varepsilon \times \mathrm{E}$

Table 1

Results of the three methods applied on the same quadrilateral panels.

\begin{tabular}{clll}
\hline & $\begin{array}{l}\text { Analysis } \\
\text { method }\end{array}$ & $\begin{array}{l}\text { Maximum principal } \\
\text { stress } \\
(\mathrm{MPa})\end{array}$ & $\begin{array}{l}\text { Maximum } \\
\text { deflection } \\
(\mathrm{mm})\end{array}$ \\
\hline $\begin{array}{c}\text { Simple bending }(50 \\
\mathrm{lbs}=222.4 \mathrm{~N})\end{array}$ & Theoretical & 34,24 & 19,40 \\
& $\begin{array}{l}\text { Simulations } \\
\text { Experimental }\end{array}$ & 40 & 20,57 \\
& 37 & 14,68
\end{tabular}

where $\varepsilon$ is the strain values read from Fig. 10 and $\mathrm{E}$ is the Young's Modulus of the material $\left(70 \times 10^{9} \mathrm{GPa}\right)$. By knowing the final strain values, the bending stress and the membrane stresses can be calculated as in Fig. 11.

It is observed that, the stresses on bottom surface become zero (no tension or compression) whereas the top surface works in compression. This demonstrates that, for this circumstance, the membrane stresses work in compression and decrease the tensile stresses created on the bottom surface and increase the compressive stresses on the top surface. The membrane effect works as pre-compression.

The maximum deflection value at the corner of the panel is recorded to be $95.25 \mathrm{~mm}$ (3.75") (Fig. 10). This value is determined as the deflection value that a fully tempered glass panel with this size $(915 \mathrm{~mm}$ by $915 \mathrm{~mm}$ ) can resist. This deformation value is specific to the size of this panel. In order to use this value for different sizes and for different cases, the surface curvature and the minimum radius of the deformed sheet are calculated using curvature analysis tools (Fig. 12). Gaussian curvature analysis is used, because it is a built-in analysis method for most of the 3D modelling programs and can be utilized during the design process to help automation.

The maximum Gaussian curvature is varying between -0.0000080206 and -0.0000083724 . The values are always negative as the sheet is in anticlastic curvature. The minimum radius values are recorded between $349.35^{\prime \prime}(8874 \mathrm{~mm}$ ) and 345.6" (8778 $\mathrm{mm})$. These can be taken as the limits for the deformation of a fully tempered glass sheet with this size

\subsection{Parameterization}

The maximum deformation and the limiting curvature occurring on the specific panel are determined for a given size of panel with a certain material. However, this does not provide much information in more generalized cases such as the cases where the size of the panel changes. With the following parameterization study, a wider database of curvature values can be obtained.

Non-planarity can be quantified by examining the surface curvature of the panel. Surface curvature, and thus non-planarity, depends on the elastic properties of the material (deformation capacity, E), the sizes of the panel ( $\mathrm{L}$ and $\mathrm{t}$ ), and the strength of the material (Fig. 13).

For the parameterization, the material selection is not taken into consideration as the properties of each material differ widely and 


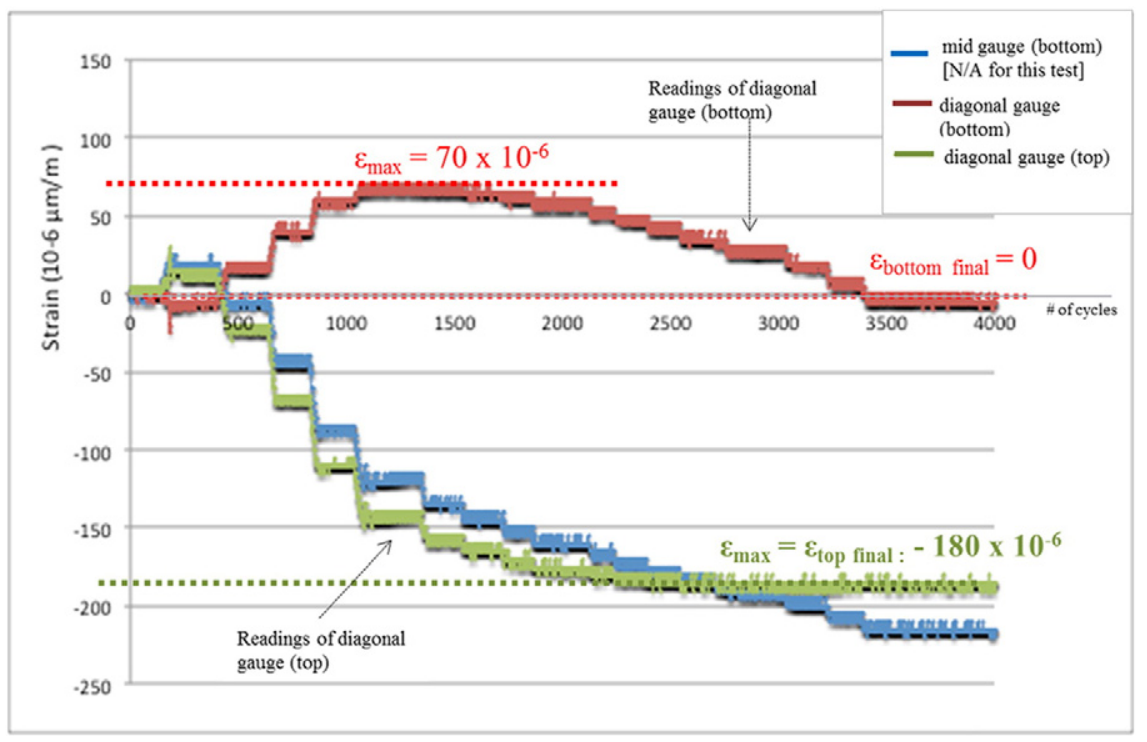

\begin{tabular}{|c|c|}
\hline \multicolumn{2}{|c|}{ Glass pre-deformation } \\
\hline load (lbs) & $\begin{array}{c}\text { Cumulative } \\
\text { deflection (inch) }\end{array}$ \\
\hline 0 & 0.00 \\
\hline selfweight & 0.50 \\
\hline selfweight & 1.00 \\
\hline selfweight & 1.50 \\
\hline selfweight & 2.00 \\
\hline selfweight & 2.20 \\
\hline sw+1 lbs & 2.25 \\
\hline sw+2 lbs & 2.31 \\
\hline$s w+3 \mathrm{lbs}$ & 2.44 \\
\hline$s w+4 \mathrm{lbs}$ & 2.53 \\
\hline$s w+5 \mathrm{lbs}$ & 2.56 \\
\hline$s w+6 \mathrm{lbs}$ & 3.00 \\
\hline$s w+7 \mathrm{lbs}$ & 3.06 \\
\hline$s w+8 \mathrm{lbs}$ & 3.13 \\
\hline$s w+9 \mathrm{lbs}$ & 3.25 \\
\hline$s w+10 \mathrm{lbs}$ & 3.56 \\
\hline$s w+11 \mathrm{lbs})$ & 3.75 \\
\hline
\end{tabular}

Fig. 10. The time history plot for the warping test.



Fig. 11. Surface stresses on the deformed glass sheet.

cannot be linearly formulated. Additionally, strength of the material is an inherited property for the material and cannot be changed. Therefore, strength is not included into the parameterization. Therefore, the relationship to be formed for the surface curvature should be dependent on the size of the panel (edge length and thickness) and the generated deformation.

Using simple geometric rules, the relationship between deformation and the surface curvature is determined to be linear for small bending values as shown in Fig. 14.

For the derivation of the relationship between the sizes of the panel and the deformation, Roark's formula for a typical rectangular panel supported on 2 adjacent sides with the two other edges free and uniformly loaded is used as a base [20]:

$\Delta_{\max }=\frac{\alpha \times q \times b^{4}}{E \times t^{3}}$

where; $\Delta_{\max }$ : Maximum deformation.

$\alpha$ : a constant value dependent on $\mathrm{a} / \mathrm{b}$ (Table 11.4 in Youngs and Budynes, [20])

b: longer edge a: shorter edge

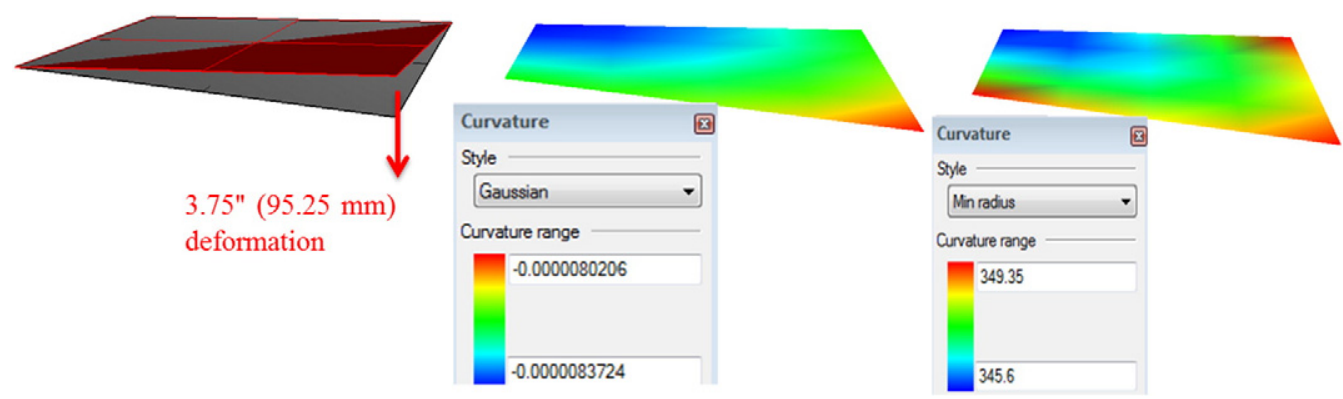

Fig. 12. Gaussian curvature and minimum radius analyses for the deformed sheet. 


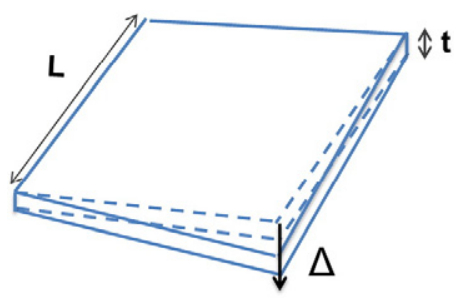

Curvature $\propto \mathrm{f}(\Delta) \propto \mathrm{f}(\mathrm{L}, \mathrm{t}, \mathrm{E})$

$\Delta:$ Deformation

L: EdgeLength

$\mathrm{t}$ : Thickness

E:Young'sModulus

Fig. 13. Design parameters for a deformed panel.

q: load per unit area t: thickness of the sheet

E: Young's modulus

assuming:

- The plate is flat with straight boundaries and constant thickness.

- Thickness is not more than one quarter of the least transverse dimension.

- The material is a homogenous isotropic material.

Then, the relationship for a 2 adjacent-edge fixed panel is obtained as follows:

$$
\begin{aligned}
& \Delta_{\max } \propto \frac{\alpha \times q \times b^{4}}{E \times t^{3}} \rightarrow \Delta_{\max } \alpha \frac{\alpha \times \frac{P}{a \times b} \times b^{4}}{E \times t^{3}} \\
& \rightarrow \Delta_{\max } \alpha \frac{\alpha \times P \times b^{3}}{E \times a \times t^{3}}
\end{aligned}
$$

This relationship demonstrates that there is an inverse proportion between the maximum deformation and the cube of thickness of the panel. There is a direct proportion between the maximum deformation and the cube of the longer edge size of the panel, whereas there is an inverse proportion between deformation and shorter edge. When the two relationships (Eq. (1) and Eq. (2)) are integrated, one parametric equation that covers all the design parameters can be written as:

Curvature $\propto \Delta_{\max } \propto \frac{\alpha \times P \times b^{3}}{E \times a \times t^{3}}$

By obtaining a parametric relationship of the variables as Eq. (3), limiting curvature value for any size of panels can be calculated when the limit of non-planarity is determined for a certain size panel for that specific material.

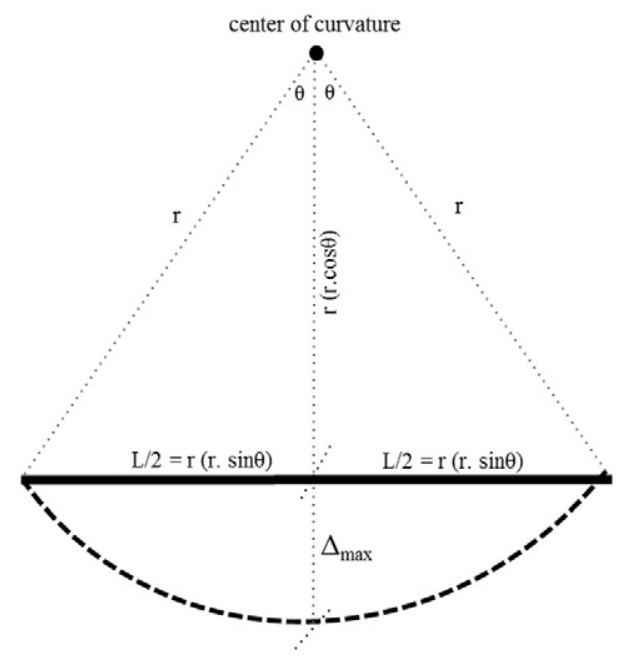

\subsection{Application}

Freeform surfaces have become more common with the help of advanced digital tools. The technology does not only help the design process, but there have been numerous digital analyses conducted, such as structural, economical, material availability, lighting, heating, etc. to control the feasibility of the design.

The proposal for this study is that the "planarity analysis" should also be part of the design of freeform surfaces, as the results affect the construction feasibility dramatically. As proposed in this study, any material's capacity for warping could be determined. (A future study could be conducted for a wider database). Then, during the discretization method, the panels can be analysed for their planarity and the curvature values can be compared with the warping limits of the selected material. If the values are within the limits, the existing non-planarity is accepted, whereas if not, then either the material selection will be reconsidered or the surface discretization will be remodelled. In any case, the capacity of the material is used as a design parameter that would help the process at an early stage of design in addition to provide the constructability of the structure.

\section{Results and conclusion}

This study focuses on the feasibility of quadrilateral paneling on freeform surface discretization. With the analyses conducted, both computer simulations and physical experiments, the method of determining the limiting values of surface curvature is demonstrated for a specific quadrilateral glass panel. In order to utilize this value in various designs, the parameterization of design variables is studied and a mathematical relationship (Eq. (3)) is established to calculate the limiting values for different sizes of panels. This process and the obtained results demonstrate that:

- Heat-tempered glass panels can be bent with a pre-determined amount, which then allows the use of partially non-planar panels on freeform surface discretization. The use of non-planarity for some of the quadrilateral panels reduces the discretization problem of free-form surfaces. By knowing the limiting curvature value of the selected material in the early stage of the design, non-planarity can be checked and necessary modifications can be done at that level in order to prevent some of the problems of construction.

- The warping of quadrilateral panels is observed to generate membrane stresses that work in advantage for the use of warped panels on the discretization of the free-form surfaces. However, not enough study has been conducted to derive a more concrete result.

$$
\begin{aligned}
& \Delta_{\max }=\mathrm{r}-\mathrm{r}(\cos \vartheta) \\
& \text { [For small } \theta \Rightarrow \cos \vartheta \cong 1-\frac{\vartheta^{2}}{2} \\
& \sin \vartheta=\frac{L / 2}{r} \cong \vartheta \\
& \Delta_{\max }=r-r\left(1-\frac{\vartheta^{2}}{2}\right) \\
& \Delta_{\max }=r-r\left(1-\frac{(L / 2 r)^{2}}{2}\right)=\frac{L^{2}}{8 r} \\
& \Delta_{\max }=\frac{L^{2}}{8} \times \frac{1}{r} \\
& \text { Curvature } \propto \frac{1}{\mathrm{r}} \quad \square \Delta_{\max } \propto \text { Curvature }
\end{aligned}
$$

(Equation 1)

Fig. 14. The relationship of surface curvature to deflection. 
Therefore this aspect of this study can be examined more deeply in future studies to conclude with more certain results.

- Non-planarity of panels, i.e. surface curvature, is dependent on numerous design variables, such as mesh sizing, the size of the panels and the material properties.

This study is conducted with one material, and demonstrates how material properties can affect the design process and the efficiency of the final product. With more analyses conducted on different materials, a database can be generated for the limits of non-planarity of numerous materials that can be used during the design process, including preliminary and final design stages. Eventually, non-planarity may become one of the analyses to be conducted during the design process of freeform surfaces. The focus of this study is not limited with the use of non-planarity of quadrilateral panels, but it also underlines the importance and efficiency of integrated design, by considering the material properties and the material-form relationship at the early stage of design.

\section{References}

[1] D. Hambleton, C. Howes, J. Hendricks, J. Kooymans, Study of panelization techniques to inform freeform architecture, architectural challenges and solutions, Glass Performance Days 2009, pp. 239-243 (ISBN 952-91-8674-6).

[2] J. Chilton, The Engineer's Contribution to Contemporary Architecture: Heinz Isler, Thomas Telford Publishing, London, 2000 (ISBN: 978-0727728784).

[3] M. Patterson, Structural Glass Facades and Enclosures, John Wiley \& Sons, New Jersey, 2011 (ISBN: 978-0-470-50243-3).

[4] C. Douthe, O. Bavenel, J.F. Caron, Form-finding of grid shell in composite materials, J. Int. Assoc. Shell Spat. Struct. (IASS) 47 (1) (2006) 53-62 April n. 150. (ISSN: 19969015).

[5] J. Schlaich, R. Bergermann, Light Structures, Prestel, Munich, 2003 (ISBN: 9783791329185).
[6] A. Holgate, The Art of Structural Engineering: The Work of Schlaich and His Team, Axel Menges, 2007 (ISBN: 978-3930698677).

[7] G. Nordenson, in: T. Riley (Ed.), Seven Structural Engineers: The Felix Candela Lectures, Museum of Modern Art, New York, 2008 (ISBN: 978-0870707032).

[8] H. Pottmann, A. Asperl, M. Hofer, A. Kilian, D. Bentley, Architectural Geometry, Bentley Institute Press, Exton, 2007 (ISBN: 978-1934493045).

[9] P. Alliez, D. Cohen-Steiner, O. Devillers, B. Levy, M. Desbrun, Anisotropic polygonal remeshing, ACM Trans. Graph. 22 (3) (2003) 485-493, http://dx.doi.org/10.1145/ 1201775.882296

[10] M. Marinov, L. Kobbelt, Direct anisotropic quad-dominant remeshing, Proceeding of PG 2004, 12th Pacific Conference on Computer Graphics and Applications 2004 pp. 207-216 (http://doi.org/10.1109/PCCGA.2004.1348351).

[11] Y. Liu, W. Xu, J. Wang, Z. Lifeng, B. Guo, F. Chen, G. Wang, General Planar Quadrilateral Mesh Design Using Conjugate Direction Field, ACM Transactions on Graphics, 30, 20116 (Article No:140. http://doi.org/10.1145/2024156.2024174).

[12] Y. Liu, H. Pottmann, J. Wallner, Y. Yang, W. Wang, Geometric modeling with conical meshes and developable surfaces, ACM Trans. Graph. 25 (3) (2006) 681-689, http:// dx.doi.org/10.1145/1179352.1141941.

[13] J. Glymph, D. Shelden, C. Ceccato, J. Mussel, H. Schober, A parametric strategy for free-form glass structures using quadrilateral planar faces, Autom. Constr. 13 (2004) (2004) 187-202, http://dx.doi.org/10.1016/j.autcon.2003.09.008.

[14] A. Berk, A Structural Basis for Surface Discretization of Free Form Structures: Integration of Geometry, Materials and Fabrication(PhD Dissertation) University of Michigan, Ann Arbor, 2012.

[15] G.H. Evolute, Evolute 2.0: EvoluteTools Pro, 2012.

[16] M. Zadravec, A. Schiftner, J. Wallner, Designing quad-dominant meshes with planar faces, Comput. Graphics Forum 29 (5) (2010) 1671-1679, http://dx.doi.org/10. 1111/j.1467-8659.2010.01776.x.

[17] A. Vaxman, A projective framework for polyhedral mesh modeling, Comput. Graphics Forum 33 (8) (2014) 121-131, http://dx.doi.org/10.1111/cgf.12405.

[18] C. Tang, X. Sun, A. Gomes, J. Wallner, H. Pottmann, Form-finding with polyhedra meshes made simple, ACM Trans. Graph. 33 (2014) 4 (Article No:70) 10.1145/ 2601097.2601213.

[19] The Institution of Structural Engineering, Structural Use of Glass in Buildings, Seto, London, 1999 (ISBN: 978-1-906335-25-0).

[20] W. Young, R. Budynas, Roark's Formulas for Stress and Strain, McGraw-Hill, New York, 1989 502-520 (ISBN: 978-0070725416). 